\title{
Digital Image Elasto-Tomography: Combinatorial and Hybrid Optimization Algorithms for Shape-Based Elastic Property Reconstruction
}

\author{
Ashton Peters, J. Geoffrey Chase, and Elijah E. W. Van Houten*
}

\begin{abstract}
Results from the application of three nonlinear stiffness reconstruction algorithms to two simple cylindrical geometries are presented in this paper. Finite-element simulated harmonic motion data with added noise were initially used to represent a measured surface displacement dataset for each geometry. This motion was used as input to gradient-descent, combinatorial optimization, and hybrid reconstruction algorithms that aimed to reconstruct two shape-based parameters describing the internal stiffness of the geometry. Both the combinatorial optimization and hybrid algorithms showed significant advantages in reconstructed parameter accuracy when compared with the traditional gradientdescent approach, with success metrics improving by $13 \%-28 \%$. Results from the hybrid algorithm applied to silicone phantom displacements demonstrated for the first time the ability of this type of algorithm to reconstruct internal stiffness using only experimentally measured surface motion data. Improvements in the sophistication of the hybrid approach should lead to improved accuracy in reconstructed solutions, as well as enabling reconstructions where the geometry is less straightforward.
\end{abstract}

Index Terms-Biomedical imaging, combinatorial mathematics, finite-element methods, inverse problems.

\section{INTRODUCTION}

C ANCEROUS breast tissue appears significantly stiffer in compression than the healthy breast tissue. This difference is due in part to the density and nature of its cellular structure, with studies showing a contrast of up to an order of magnitude difference in Young's modulus [1]-[3]. The ability of manual palpation to detect abnormal growths within the breast is a direct result of this stiffness contrast.

Elastographic techniques aim to image and quantify the elastic properties of human tissue. The mechanical stiffness contrast measured with elastographic methods is far greater than the $\mathrm{X}$-ray attenuation contrast measured with a screen-film mammogram, which is regarded as the gold standard for breast cancer screening [4]-[6]. Several groups have had success in reconstructing the elastic properties of the breast volume using different elastographic methods, particularly MR elastography and

Manuscript received October 4, 2007; revised February 6, 2008. First published June 10, 2008; current version published October 31, 2008. Asterisk indicates corresponding author.

A. Peters is with Boundary Lifesciences, Inc., Christchurch 8140, New Zealand, and also with the Department of Mechanical Engineering, University of Canterbury, Christchurch 8140, New Zealand (e-mail: ashton.peters@canterbury.ac.nz).

J. G. Chase is with the Department of Mechanical Engineering, University of Canterbury, Christchurch 8140, New Zealand.

*E. E. W. Van Houten is with the Department of Mechanical Engineering, University of Canterbury, Christchurch 8140, New Zealand (e-mail: elijah.vanhouten@canterbury.ac.nz).

Digital Object Identifier 10.1109/TBME.2008.2001132 ultrasound elastography [7]-[10]. Both techniques have yielded comparable results to direct mechanical measurement of tissue. Sensitivity and specificity figures for elastography are only now being calculated through robust clinical trials. As such, data for direct comparison with mammography are not yet available.

Digital-image-based elasto-tomography (DIET) is a novel soft tissue elastography method that uses surface motion as input to an inverse reconstruction algorithm that calculates the internal stiffness distribution of a harmonically exited object. This reconstruction problem has less input data, and therefore, possesses a greater degree of ill-posedness than other modalities, such as MR elastography, where full-volume datasets are available. However, the ability to obtain data via inexpensive digital imaging technology makes the approach practically and clinically attractive. In addition, it may offer other advantages in portability, speed of testing, and elimination of X-ray dose. An outline of the major steps in the DIET process are as follows.

1) A steady-state sinusoidal motion is induced throughout an object volume by an actuator placed against its surface.

2) Spatially calibrated digital imaging sensors arrayed around the object capture sequential 2-D images of reference points on the surface of the object as they cycle through the full range of steady-state motion.

3) Image processing algorithms convert consecutive sets of 2-D images of reference points on the surface into a harmonic 3-D vector description of the point motion containing amplitude and phase information.

4) The vector description of each reference point's motion is used in an inverse reconstruction algorithm that generates an elastic modulus distribution within the 3-D object volume.

Combinatorial optimization (CO) is a stochastic technique that involves efficiently exploring problem search domains to solve large and complex nonlinear optimization problems faster than an exhaustive search [11]. Applications of CO in the field of elastic property reconstruction are limited, though increasing in popularity as more powerful computer systems become mainstream. Matsuoka et al. used a genetic algorithm (GA) in combination with finite element (FE) methods to solve for the mechanical properties and internal structure of composite materials. Here, the inverse algorithm optimized the internal structure of a composite to obtain a desired Young's modulus value [12]. Olmi et al. performed a preliminary investigation into the use of a GA for image reconstruction in electrical impedance tomography (EIT). A numerical model was used to simulate the electrical properties of a simple 
geometry containing a high-resistivity inclusion. While the GA used in this study to perform image reconstruction was found to have significantly higher computational cost than more traditional Newton-Raphson methods, advances in computer speed were seen as a pathway to the possible implementation of such optimization techniques in real-time EIT systems [13].

The use of stochastic techniques in soft tissue elastography is a new and emerging field. Zhang et al. developed a theoretical framework for the application of a GA to problems in soft tissue elastography. To ensure numerical stability, this method constrained solutions using an assumed a priori property distribution. Simulating displacements with a synthetic numerical model, stochastic methods were found to give consistently accurate results for the elastographic reconstruction problem [14]. Khalil et al. developed a combined GA and FE approach to solve for elasticity values in vascular soft tissue. This study lumped material parameters into discrete regions in order to simplify the genetic encoding process and used a numerical model to simulate displacements in a 2-D model. Successful elastic property reconstructions were performed on both homogeneous and heterogeneous models with multiple inclusions in the presence of noise [15].

Though novel reconstruction techniques based on a stochastic approach, such as GAs, have been applied to inverse problems where the FE method is used for system simulation, the application of such methods to the tomographic elastography problem has not been considered, particularly when using only exterior surface motion measurements from experimental phantoms. Proof of concept studies for the DIET system, including a first experimental proof of concept evaluation using experimental data, have been previously published [16], [17]. The research presented here describes a shape-based reconstruction approach applied to both FE-simulated and phantom displacements, where both deterministic and stochastic reconstruction approaches are applied to simple heterogeneous geometries.

\section{METHODS}

Three inverse reconstruction algorithms were used to solve for the internal Young's modulus distribution of two different phantom geometries. The first stage of the experiment involved collecting surface motion data. This was obtained first using the FE simulation described in Section II-A, and then, experimentally from silicone phantoms described in Section II-B. Section II-C-F describes the three inverse reconstruction algorithms that are evaluated using both simulated and experimentally measured surface motion data. Reconstruction of an internal stiffness distribution was performed via two shapebased parameters describing an interface position and a stiffness contrast between the hard and soft material. The shape-based parameterization approach was chosen as it allows a large variation in internal stiffness distribution to be modeled using only two parameters. While this two-parameter approach would not be appropriate in a clinical scenario for breast lesion detection, it is sufficient to allow testing of reconstruction algorithms at this early stage. A clinical implementation of this approach would require an increased number of parameters to effectively

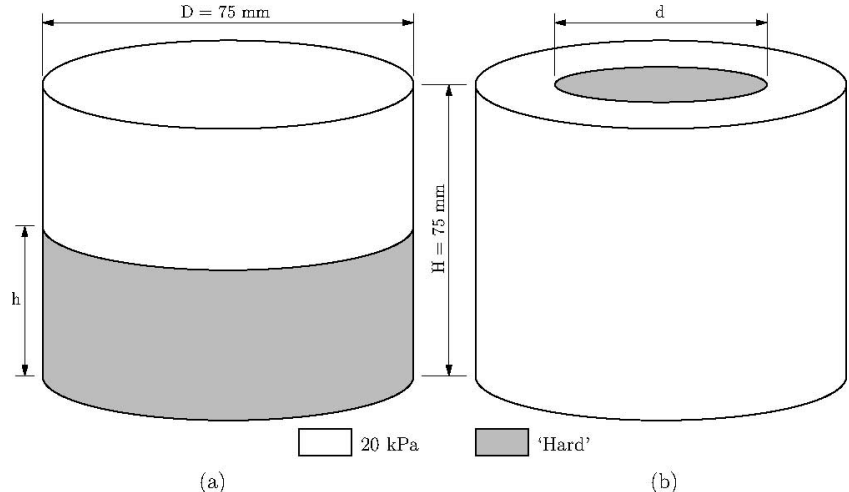

Fig. 1. Geometry of the two phantoms in this experiment. (a) Stacked geometry where the cylinder is divided vertically into two regions with hard material below the soft material. (b) Concentric geometry where a hard vertical cylinder is contained within a larger soft cylinder.

describe the more complicated internal geometry expected in such cases. The overall reconstruction approach would remain similar to that presented here; however, increasing reconstructed parameters would lead to an increased computational demand.

An undamped material model was used to reduce the number of parameters required for reconstruction [18]. This model was found to be adequate for testing reconstruction algorithms using both simulated data and experimental data from silicone phantoms. For consistency within this experiment, the same undamped model was used to perform reconstructions on the experimental data. While such a model may not provide an exact match to in vivo tissue behavior, it was considered sufficient for this preliminary study. Clinical applications in vivo will require damping characterization that is readily included through the use of a complex shear modulus in this time-harmonic displacement case.

\section{A. Finite-Element Simulation}

Surface motion datasets required for the simulation study were generated using FE software written in Fortran 90. Two cylindrical phantoms composed of distinct hard and soft regions were considered, with the specific geometries shown in Fig. 1. The overall phantom dimensions were chosen to roughly approximate the size order of a female breast, the intended target of the final DIET system.

The distribution of Young's modulus $E$ throughout each geometry was described by two independent model parameters. The interface position $P$ between hard and soft material was defined for the stacked geometry of Fig. 1(a) as $h / H$ and $d / D$ for the concentric geometry of Fig. 1(b). In both cases, $H=$ $D=75 \mathrm{~mm}$.

The stiffness contrast $C$ was defined for both simulated models as $E_{h} / E_{s}$. The value for $E_{s}$ was fixed at $20 \mathrm{kPa}$, a value chosen to be of the same order as Young's modulus measurements of fat and glandular tissues in the human breast [18]. Poisson's ratio $\nu$ and density $\rho$ were fixed at 0.45 and $1000 \mathrm{~kg} / \mathrm{m}^{3}$, respectively, approximating the nearly incompressible nature and high water content of human tissue. 
TABLE I

PARAMETER VALUES REPRESENTING REAL DATA FOR SIMULATION STUDIES

\begin{tabular}{ccc}
\hline Geometry & Interface Position & Stiffness Contrast \\
& $P_{0}$ & $C_{0}$ \\
\hline Stacked & $40 \%$ & $400 \%$ \\
Concentric & $50 \%$ & $300 \%$ \\
\hline $\begin{array}{l}\text { The corresponding motion datasets where generated by adding noise to FE } \\
\text { simulated motion at these parameter values. }\end{array}$
\end{tabular}

An FE mesh containing approximately 169000 linear tetrahedral elements and 31000 nodes was created using the preprocessing software Gambit. The average element edge length was $3 \mathrm{~mm}$, which represented a displacement converged mesh with relative displacement error of less than $4 \%$. The bottom face of the cylinder was constrained in the horizontal plane and actuated vertically with an amplitude of $1 \mathrm{~mm}$ at a frequency of $50 \mathrm{~Hz}$. Both phantom geometries were simulated using the same mesh, with Young's modulus values assigned nodally based on the geometric location within the cylinder and the specified values of $P$ and $C$ for a given case.

The target parameters $P_{0}$ and $C_{0}$ that represented the real dataset for each geometry are shown in Table I. All 3215 nodes on the vertical exterior of the cylinder were chosen as reference points where the motion was measured. This sampling method provided a simple approximation to the final DIET system, where motion data from the full surface will be available. Noise added to the simulated real datasets was normally distributed with a mean of zero and a variance of $10 \%$ of the average reference point displacement amplitude.

FE simulation was also used to generate a motion database for both geometries across a range of values for $P$ and $C$. Simulations were performed at interface positions $P$ ranging from $10 \%$ to $90 \%$ with increments of $1 \%$, and stiffness contrasts $C$ from $100 \%$ to $500 \%$ with increments of 5\%. These parameter limits defined the problem domain for all reconstruction algorithms. While a small systematic error is introduced when discretizing the parameter domain in this manner, the error was considered insignificant, given the limited accuracy required at this early stage of research. Contrast values were chosen to give a conservative range based on independent measurements of ex vivo tissue stiffness [1], [2]. A total of 6561 simulations for each geometry were performed on a workstation with dual Advanced Micro Devices (AMD) Opteron processors using the Multifrontal Massively Parallel Sparse (MUMPS) matrix solver [19]. Each FE simulation required approximately 100 seconds. This database of simulations allowed the evaluation of error over the problem domain prior to attempting reconstructions, reducing the time taken to perform parameter reconstruction to less than $1 \mathrm{~s}$ per iteration.

\section{B. Experimental Phantom Data}

Experimental motion data were generated from two silicone phantoms created with the same geometry as the simulated models. Fig. 2 shows the experimental setup of the actuator, dual cameras, and strobe for lighting, as well as the two silicone phantoms used for motion capture. Camera calibration was per-

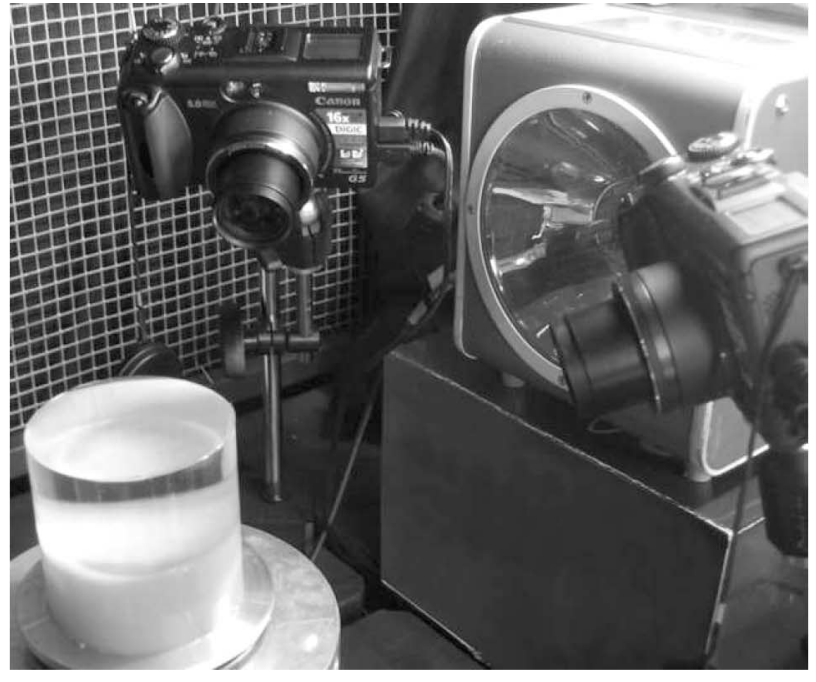

(a)

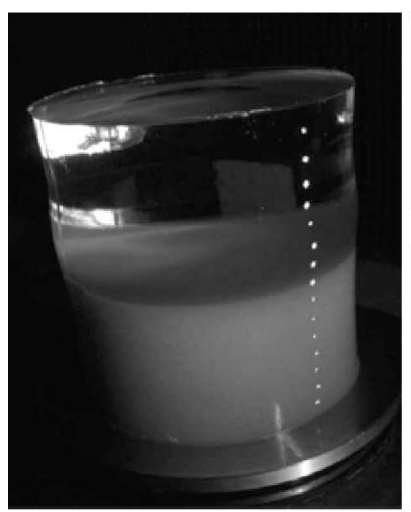

(b)

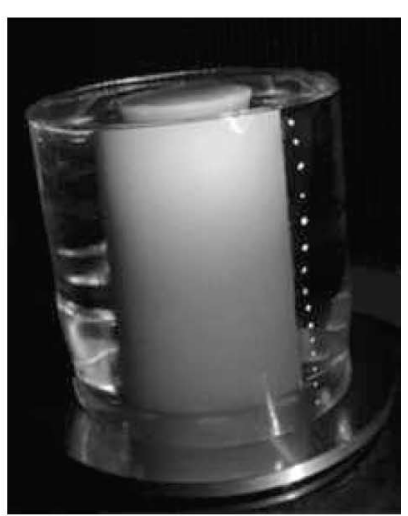

(c)
Fig. 2. Experimental setup and silicone phantoms used for experimental parameter reconstruction, where the surface reference points used for motion comparison are visible. (a) Experimental setup. (b) Stacked phantom. (c) Concentric phantom.

formed using standard techniques outlined in more detail in [16]. Harmonic motion data were collected and processed from these phantoms using an experimental setup previously described for homogeneous gelatine phantoms [17], [20], where actuation was at $100 \mathrm{~Hz}, 0.5 \mathrm{~mm}$ amplitude.

A column of 15 small dots on the vertical exterior of the phantoms formed the reference points used for motion tracking and comparison in the experimental cases. The use of tightly controlled actuator position and strobe lighting allowed a sequence of 20 images of the phantom surface to be taken with each camera. Calibration and measurement error arising from image capture and processing has been characterized at a level of $\pm 1-2$ pixels, corresponding to a position error of $0.1-0.2 \mathrm{~mm}$. Any adverse affects of this error are minimized when considering that this error is approximately constant for small regions of space. Using the known calibration information from each camera, the 2-D position of each reference point was converted into a 3-D position in a fixed laboratory reference frame, from which comparison with FE simulation could take place. Previous static mechanical property tests and analysis of homogenous phantoms have indicated that the soft and hard silicone materials 


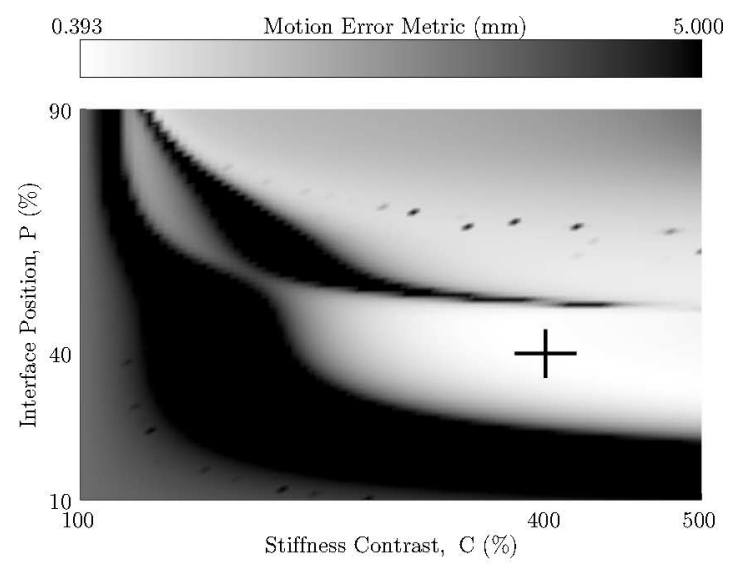

(a)

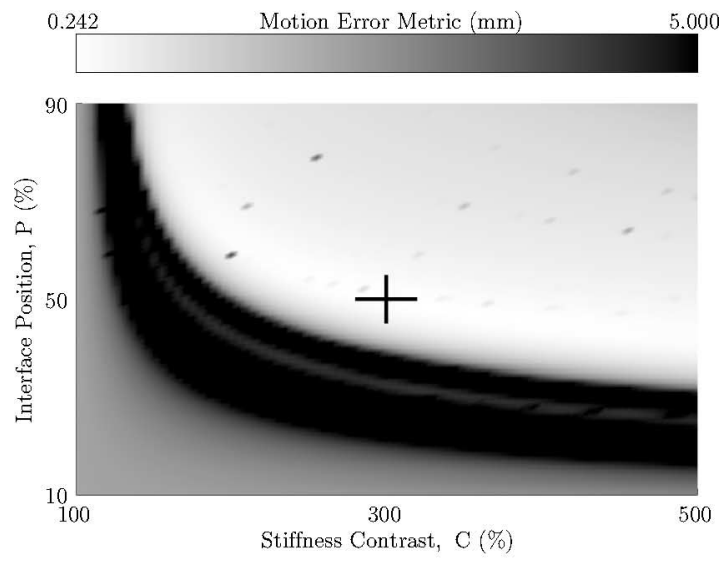

(b)

Fig. 3. Displacement error metric defined in (1), evaluated across the domain of interest for both geometries. In both cases, the black cross indicates the target parameter combination. The mean surface displacement in each case was 1.9 and $1.5 \mathrm{~mm}$, respectively. The average motion error metric has an upper limit of $5 \mathrm{~mm}$ in these plots. Noise was added to the target data before the metric was calculated. (a) Stacked geometry. (b) Concentric geometry.

have Young's modulus values of $25-30 \mathrm{kPa}$ and $100-130 \mathrm{kPa}$, respectively [21].

\section{Parameter Reconstruction}

All three nonlinear parameter reconstruction algorithms presented minimize the squared error between a set of "measured" displacements at the target parameters $y$ and displacements calculated based on current estimates of parameters $P$ and $C, f(\theta)$, where $\theta$ represents the combination of both $P$ and $C$. The error metric $\Omega$ represents the mean squared motion error across all reference points, and is defined as

$$
\Omega=\sqrt{\frac{\sum_{i=1}^{N}\left\|y_{i}-f(\theta)_{i}\right\|^{2}}{3 N}}
$$

where $N$ is the number of reference points. This error metric formed the cost function that all reconstructions were performed against.

Fig. 3 shows the error metric from (1) calculated across the problem domain, where the "measured" displacements were

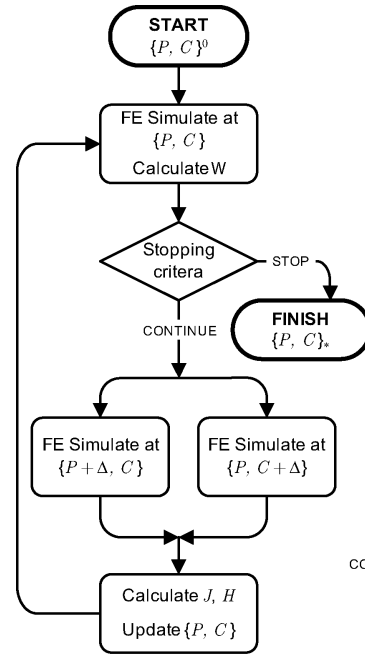

(a)

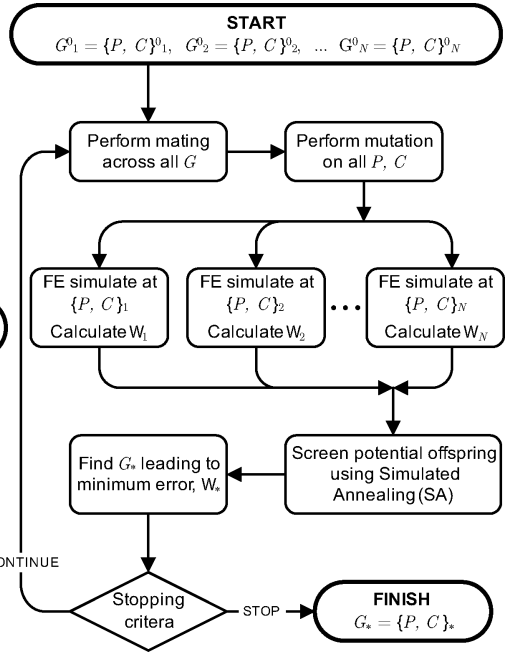

(b)
Fig. 4. Schematics of the GD and CO algorithms used for parameter reconstruction. (a) GD. (b) CO.

those simulated at the target parameters shown in Table I, with the addition of noise. The minimum error value for the stacked geometry is $0.39 \mathrm{~mm}$, and for the concentric geometry is $0.24 \mathrm{~mm}$. The cross-hair in the figures shows the target parameter combination, which corresponds to the minimum error metric in both cases. This error information provided both data and context to the reconstruction algorithms tested subsequently.

Parameter updates for $P$ and $C$ within all algorithms were rounded to the nearest $1 \%$ and $5 \%$, respectively. Additionally, the value of $P$ was constrained between $10 \%$ and $90 \%$, and $C$ between $100 \%$ and $500 \%$, restricting all reconstructions to the problem domain bounding the target parameter combination.

\section{Gradient-Descent Algorithm}

The gradient-descent (GD) algorithm used for parameter reconstruction was based on the Gauss-Newton method, and is described in Fig. 4(a). For the isotropic case, where $\nu$ and $\rho$ were assumed to be known and constant throughout, only $P$ and $C$ required reconstruction. The full Gauss-Newton iterative formulation is developed in more detail in [16], and results in the equation

$$
\theta_{r+1}=\theta_{r}+\delta_{r}\left(\left(\frac{\partial f}{\partial \theta}\right)^{T}\left(\frac{\partial f}{\partial \theta}\right)\right)^{-1}\left[\left(\frac{\partial f}{\partial \theta}\right)^{T}(y-f(\theta))\right]
$$

where $r$ represents the current iteration and $\delta$ is chosen to influence the step size of the algorithm. In this case, the approximate Hessian matrix $(\partial f / \partial \theta)^{T}(\partial f / \partial \theta)$ was not regularized for stability due to the small number of unknown parameters compared with the number of known surface motions.

For the case where reconstructed parameters are not nodally distributed and are independent of the FE mesh used for simulation, the Jacobian matrix $(\partial f / \partial \theta)$ requires rederivation each time the reconstructed parameters are changed to describe different geometry. As this process is time-consuming and the resulting Jacobian terms are geometry-specific, the Jacobian 
was generated using a finite-difference approximation in which matrix columns were approximated as

$$
J(:, i)=\frac{f\left(\theta_{i}+\Delta\right)-f\left(\theta_{i}\right)}{\Delta}
$$

where $\Delta$ represents a small change in either $P$ or $C$. This approach allows a straightforward derivative calculation regardless of the parameters and geometry used.

Reconstructions were performed with a range of initial parameter guesses across the problem domain. Initial parameter guesses were generated for $P$ every $2 \%$ between $10 \%$ and $90 \%$, and for $C$ every $10 \%$ between $110 \%$ and $490 \%$, resulting in a total of 1521 reconstructions with starting locations spanning the problem domain. A reconstruction was stopped when any of the following criteria were satisfied.

1) The surface motion error metric $\Omega$ was less than a predetermined minimum based on the minimum possible surface motion error value.

2) The algorithm step size was zero for both parameters for more than two consecutive iterations.

3) A total of 100 iterations had been performed.

The first criterion represented a definite successful reconstruction. The second was a case where the gradient was either so shallow that the algorithm halted or so steep that the reconstruction continually attempted to step outside the problem domain, and the update was ignored. The third case covers a failure to converge or to converge quickly enough.

\section{E. Combinatorial Optimization}

The $\mathrm{CO}$ algorithm implemented in this experiment utilized the population mating mechanisms provided by simple GAs with the more sophisticated selection criteria provided by simulated annealing (SA) [22], [23]. The basic structure of the algorithm is shown in Fig. 4(b) and is described in the following.

1) The population comprised ten chromosomes and a maximum of 100 generations were simulated.

2) Random initial guesses for $P$ and $C$ were assigned to all chromosomes in the population. All guesses were restricted to lie within the problem domain $10 \%<P<$ $90 \%$ and $100 \%<C<500 \%$.

3) FE simulation was performed to obtain surface motion and a surface motion error value from (1) for all chromosomes.

4) A new generation was formed by mating all chromosomes in the population using roulette wheel selection [24]. In this experiment, mating was performed by swapping binary bits between the two chromosomes involved. The probability of bits within each chromosome pair swapping was $90 \%$. Mating was not performed if it resulted in new chromosomes having parameter values outside the problem domain.

5) Random mutation was performed on all chromosomes in the new generation. Mutations were performed at bit level where the probability of a single bit within a chromosome flipping was $2 \%$. As with mating, mutation was restricted to generating parameter values within the problem domain.
6) A Metropolis scheme was used to select the chromosomes that make up the new generation [25]. The probability that a chromosome from the new generation replaced its counterpart in the previous generation was defined as

$$
\Pi_{\text {accept }}=\exp \left(\frac{-\Omega_{r} / \Omega_{r-1}}{T_{r}}\right)
$$

where $r$ is the current generation and $T$ is a systemwide temperature variable that was decreased each generation. In this experiment, the temperature was decreased logarithmically between values of 10 and 0.5 over 100 generations.

7) The error metric $\Omega$ and parameters $P$ and $C$ corresponding to the best fitting chromosome were tracked over all generations. The algorithm was stopped early if this lowest $\Omega$ value was less than a predefined limit. This indicated a successful reconstruction.

\section{F. Hybrid Algorithm}

The hybrid algorithm developed for this experiment was a combination of previously described CO and GD algorithms that aimed to combine the positive aspects of both methods. The first stage of the algorithm involved running the $\mathrm{CO}$ algorithm shown in Fig. 4(b) for 100 generations. Upon completion of $\mathrm{CO}$, the parameter set with the lowest error $G_{*}=\{P, C\}_{*}$ was used as the initial guess for a GD reconstruction that progressed according to Fig. 4(a) for up to ten iterations. The finish point of the GD reconstruction was considered the solution of the hybrid reconstruction method. As such, the hybrid algorithm was not a "ground-up" implementation, but rather a serial combination of the CO and GD algorithm, where the execution of each stage was controlled using Linux shell scripting.

\section{RESULTS}

The overall success of each type of reconstruction algorithm across all starting values for a particular geometry was represented by a cumulative, normalized parameter error value:

$$
\Psi=1-\left(\frac{\sum_{i=1}^{N}\left\|P_{0}-P_{i}\right\|}{P_{0}}+\frac{\sum_{i=1}^{N}\left\|C_{0}-C_{i}\right\|}{C_{0}}\right) / 2 N
$$

where $P_{0}$ and $C_{0}$ are the target parameters from Table I and $N$ is the total number of reconstructions performed on the geometry in question.

The distribution of GD reconstructed solutions is shown in Fig. 5(a) and (b). The success metric for the GD algorithm when applied to the stacked and concentric geometries is $71.2 \%$ and $78.2 \%$, respectively. In both cases, the majority of failed reconstructions were those that attempted to step to parameter values outside the problem domain. These reconstructions can be seen at lower left of Fig. 5(a) and upper left of Fig. 5(b). None of the GD reconstructions were stopped due to a failure to converge, indicating that a sufficient number of iterations had been performed.

To allow statistical comparison with the GD algorithm, the same number of CO reconstructions (1521) were performed on each geometry. Fig. 5(c) and (d) shows the distribution of 


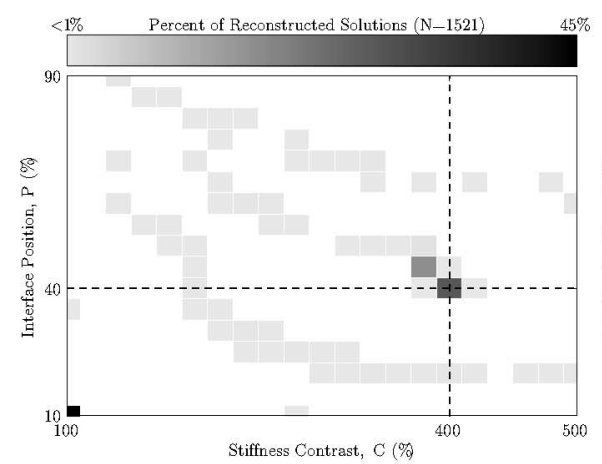

(a)

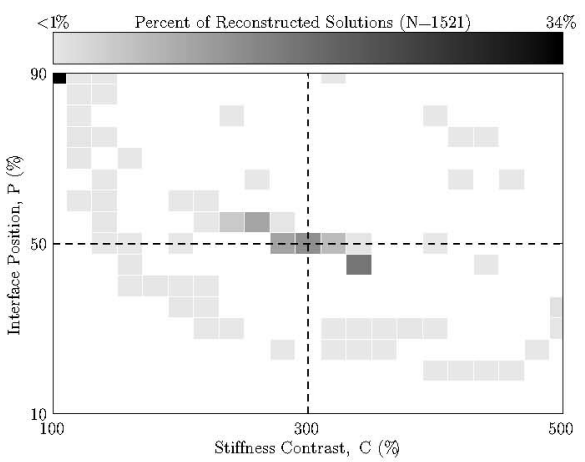

(b)

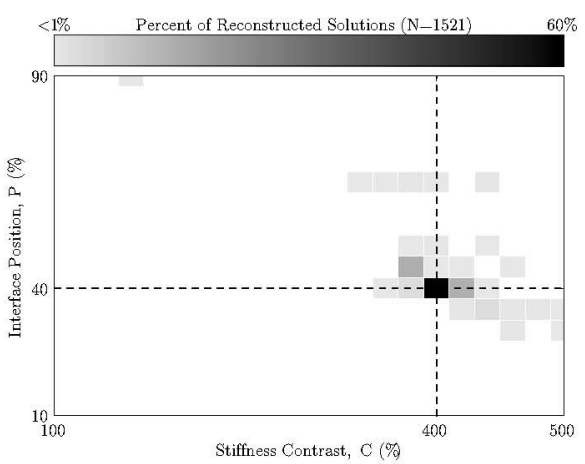

(c)

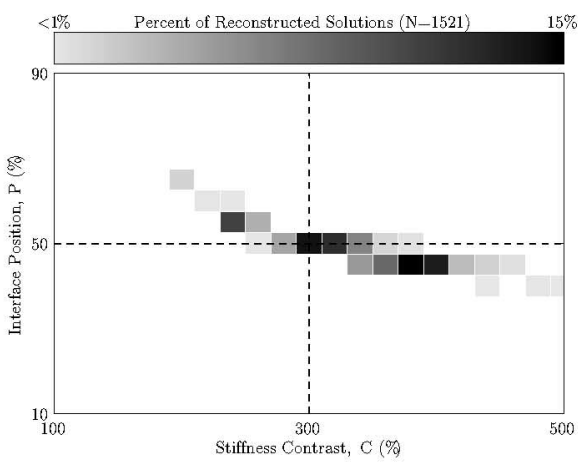

(d)

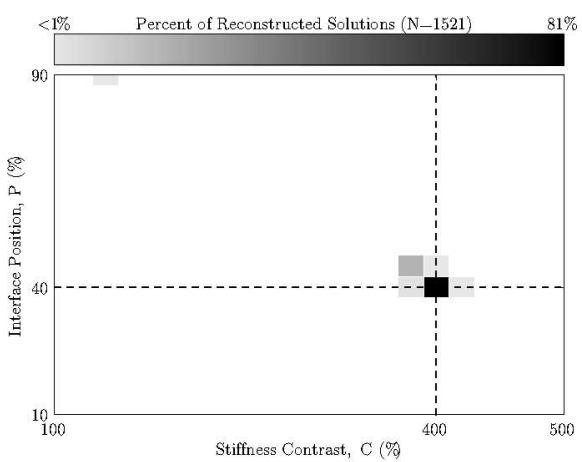

(e)

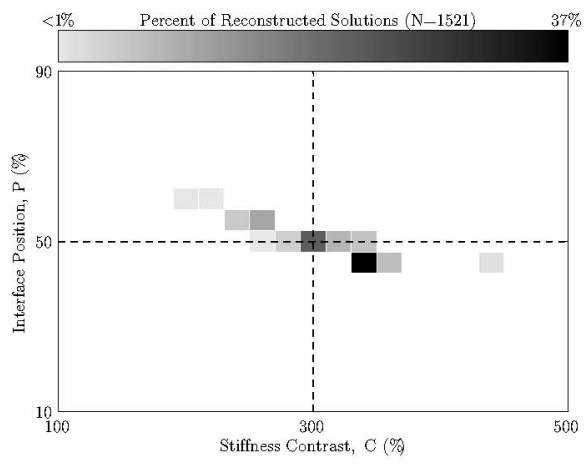

(f)

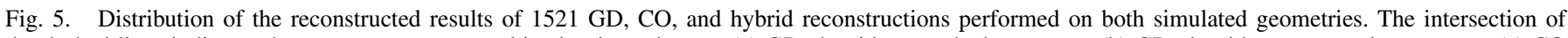
the dashed lines indicates the target parameter combination in each case. (a) GD algorithm, stacked geometry. (b) GD algorithm, concentric geometry. (c) CO algorithm, stacked geometry. (d) CO algorithm, concentric geometry. (e) Hybrid algorithm, stacked geometry. (f) Hybrid algorithm, concentric geometry.

TABLE II

SUMmaRY OF RECONSTRUCTION ALGORITHM SUCCESS METRIC $\Psi$ ACROSS BOTH STACKED AND CONCENTRIC GEOMETRIES

\begin{tabular}{cccc}
\hline Algorithm & Stacked & Concentric & Simulations \\
\hline GD & $71.2 \%$ & $78.2 \%$ & 60 \\
CO & $97.8 \%$ & $91.2 \%$ & 1000 \\
Hybrid & $98.9 \%$ & $94.2 \%$ & 1060 \\
\hline The total number of FE simulations required for each reconstruction
\end{tabular}

algorithm is also given.

reconstructed solutions. The success metric $\Psi$ for the stacked and concentric geometries is $97.8 \%$ and $91.2 \%$, respectively. Less accurate reconstructed solutions are largely confined to a smaller area or band adjacent to the target location.

The final simulated data reconstructions involved the same 1521 instances of the hybrid reconstruction, using results from the previously performed $\mathrm{CO}$ as the first phase. The reconstructed solution plots for both geometries are given in Fig. 5(e) and (f), where success metrics for the stacked and concentric geometries are $98.9 \%$ and $94.2 \%$, respectively. A clear reduction in solution spread is apparent when comparing Fig. 5(a) and (b) with Fig. 5(c) and (d) and 5(e) and (f). The success metric $\Psi$ for the three algorithms applied to simulated data across both phantom geometries are summarized in Table II.

Fig. 6 is the result of 200 instances of the hybrid reconstruction algorithm applied to the experimental data from both stacked and concentric silicone phantoms. Here, due to a higher motion error sensitivity to the soft modulus value, the stiffness contrast parameter $C$ represents the ratio $E_{s} / E_{h}$, with the hard modulus fixed at $100 \mathrm{kPa}$. Panels (a) and (b) clearly show the further reduction in error metric after iteration 20, where the hybrid algorithm switches from CO to GD, particularly in the stacked geometry case. Also evident is a reduction in the spread of the reconstructed results from iteration 20 onwards as the GD algorithm encourages convergence. The distribution of reconstructed results for the stacked geometry indicate that the interface position is well reconstructed with the stiffness contrast underestimated by 5\%-10\% (absolute). The concentric geometry results in Fig. 6(d) show a greater spread for both parameters, with both stiffness contrast and interface position slightly underestimated.

\section{DISCUSSION}

The error plots in Fig. 3 provide a clear indication of the error metric trends across the problem domain. The large areas of resonance observed are a result of the undamped linear mechanical model used for FE simulations. The stacked geometry has a more complicated resonance pattern as the geometry places the interface between hard and soft material perpendicular to the mechanical waves generated by actuation of the model. While the areas of resonance indicate simulated displacements becoming unrealistically large, they also provide a challenging problem domain with clear local minima. A large area of low error surrounds the target parameter combination for the concentric geometry, making this reconstruction problem especially difficult. 


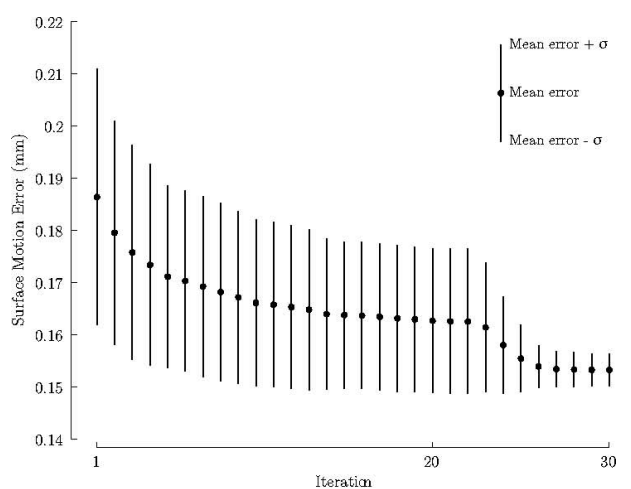

(a)

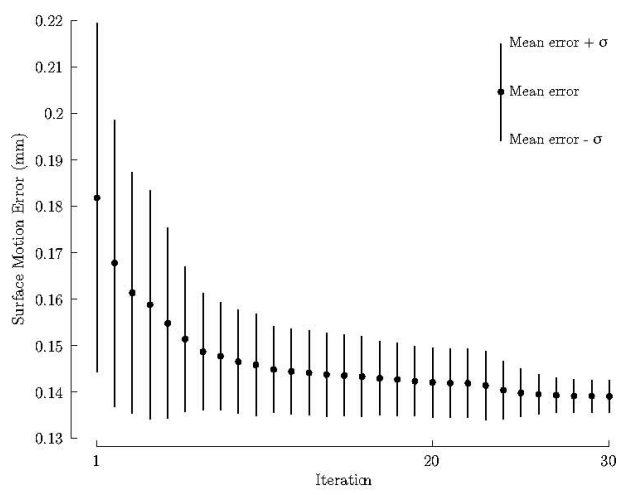

(b)

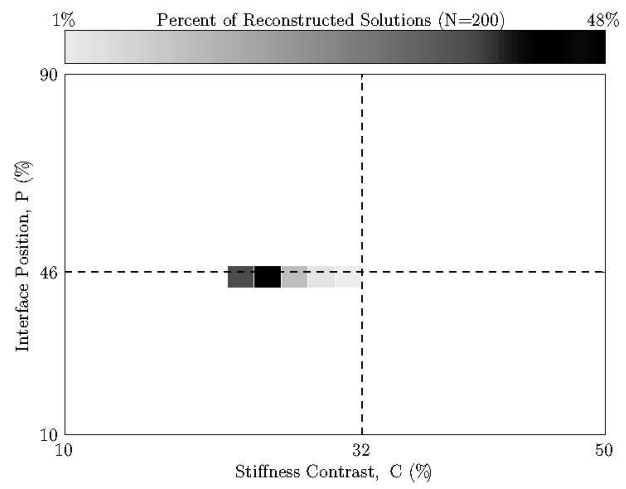

(c)

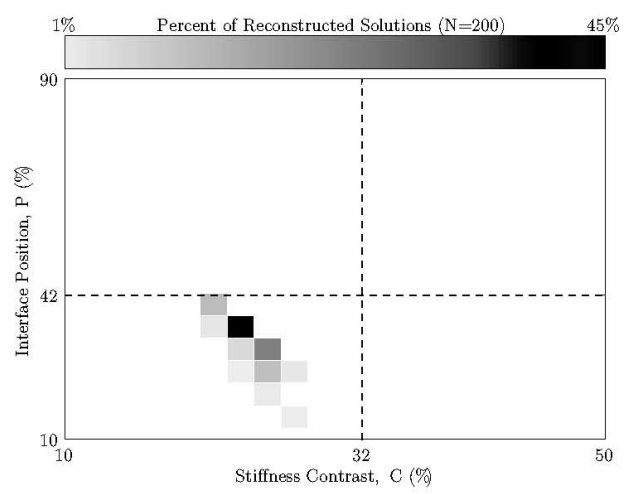

(d)

Fig. 6. Collated reconstruction algorithm progress and final reconstructed parameters from performing 200 instances of the hybrid algorithm on real motion data from both silicone phantoms. (a) Stacked geometry. (b) Concentric geometry. (c) Stacked geometry. (d) Concentric geometry.
The large spread of solutions for the GD reconstructions means a high chance that a randomly selected starting position will not lead to a successful reconstruction. Analysis of failed reconstructions showed that when started on the opposite side of a resonance band, the GD solver was not able to reconstruct the correct solution. However, the solver performed very well when started in the vicinity of the solution, supporting its use in the hybrid algorithm, given the small number of FE simulations required to obtain the solution when it succeeded.

The concentric geometry demonstrates that while $\mathrm{CO}$ is successful at avoiding local minima, its ability to differentiate between the correct solution and the one close to it is limited. This limitation constrains the algorithm's effectiveness when dealing with problems where the correct solution lies surrounded by a continuity of very similar solutions. However, a qualitative comparison of Fig. 5(b) and (d) clearly shows an obvious clustering about the correct solution for the $\mathrm{CO}$ reconstruction. Depending on the contrast required, this may be clinically acceptable, given the higher success metrics observed.

The hybrid algorithm clearly had the greatest success across both geometries in the experiment, with success metrics above $94 \%$ for both geometries. This result demonstrates the potential for a reconstruction algorithm combining the advantageous aspects of GD and CO to have markedly greater success than either algorithm in isolation. Both the hybrid and $\mathrm{CO}$ algorithms were superior to the GD algorithm in their ability to identify a single unique solution. Although both $\mathrm{CO}$ and hybrid algorithms had a spread of reconstructed results, these were clustered about the minimum error solution.

The number of forward simulations required for each reconstruction algorithm gives an estimate of the computational cost of each approach. While the $\mathrm{CO}$ and hybrid methods have significantly higher numbers of simulations required, it is important to note that a large number of these simulations are independent and could be performed in parallel across multiple processors. For the $\mathrm{CO}$ algorithm, which also formed the first 100 iterations of the hybrid method, this would reduce the time taken to complete all simulations by a factor of 10 as each generation contained ten independent chromosomes. Future work will investigate the performance of the $\mathrm{CO}$ method, given less generations in an effort to reduce its computational cost.

Reconstructions on both geometries clearly benefitted from the use of a hybrid algorithm, with the additional reduction in mean error and error spread visible in Fig. 6(a) and (b). The less significant reduction of error metric in the concentric case after iteration 20 appears to be due to the better performance of the $\mathrm{CO}$ phase of the reconstruction on this geometry. Though there is less room for improvement in this case, the addition of the GD algorithm can still be seen to reduce the mean error metric and error spread, both of which are important when considering the desired repeatability of DIET stiffness reconstructions.

The reconstructed parameter values from the experimental phantom data in Fig. 6(c) and (d) indicate a reasonable agreement with measured values for both geometries, with the stacked case showing a closer match. The measured stiffness contrast was based on static measurements from silicone samples produced at a different time to the phantoms, meaning the measured 
value was not expected to provide an exact target for reconstruction, given the number of experimental uncertainties. The reduced spread in the positional parameter for the stacked geometry reconstructions in Fig. 6(c) is likely due to the reference motion measurement being located directly on the hard and soft silicone regions, allowing the interface position to be accurately identified due to the step change in shear wavelength across the two materials. The concentric geometry had a significantly lower error sensitivity to interface position, and consequently, less accurate interface position estimates. The physical separation of the measurement locations from the stiffness interface provides evidence as to why this loss of accuracy occurs.

Improvements in the sophistication of both the GD and $\mathrm{CO}$ should lead to increased reconstruction success rates. Both algorithms are very simple implementations of these techniques, and could benefit from added features, such as regularization and more sophisticated evolution strategies. The hybrid algorithm could be improved by a closer investigation of the best combination of constituent algorithms, possibly applying both techniques simultaneously rather than in sequence.

Reducing noise is a constant priority when working with accurate experimental systems such as DIET. Increasing the image resolution beyond the two megapixel images currently used may allow for more accurate calibration and a reduction in position error. However, the increased computation required when dealing with larger images outweighed the gain possible with this approach, particularly considering other sources of experimental error that are not as easily controlled.

Adding further reference markers for motion tracking will allow more accurate characterization of the phantom surface motion during actuation. Additionally, using a regular grid or random array of markers on the phantom surface will allow any effects of asymmetry in the phantom to be observed. This approach will be taken when more realistic phantom trials are undertaken with the DIET system. In addition, using circular instead of rectangular surface markers will allow for more accurate reference point tracking, given the projective invariance of a circle's centroid when moving on the phantom surface.

The isotropic material model used for this study is likely to be in close agreement with the silicone material used for the experimental phantoms. When considering in vivo breast tissue, however, this assumption may not prove accurate, given the considerably more complicated internal structures present. However, recent elastography studies have obtained successful in vivo results using the simplified isotropic assumption [9], [18].

The match between simulated and experimental boundary conditions was observed to be very close during phantom actuation, minimizing any possible error due to a mismatch in constraint modeling. When applied in a clinical scenario, the boundary conditions used for FE simulation will need to take into account the more complicated actuator/breast interface as well as the conditions at the chest wall. The development of these conditions and their influence on both measured motion and reconstructed results will be the target of subsequent study in this field.
Future work will concentrate on repeating these results with more complicated geometries that are a better approximation to the final DIET system. In addition, a potential exists to apply statistical methods to further improve the reconstruction results and computational efficiency. Such statistical methods might allow the combination of several reconstruction runs into a pool of global reconstructions, from which metrics such as the mean and variance can give an indication not only of the reconstructed result, but the confidence in such a result. These techniques would, however, increase the computational cost over a single reconstruction when performed on a serial system due to the large number of independent reconstructions required. However, parallel systems provide a potential method for reducing the total time needed.

\section{CONCLUSION}

The investigation performed shows that the shape-based reconstruction is able to identify both a positional and a stiffness contrast parameter for varying physical geometries in both simulation and phantom studies. Of the three reconstruction algorithms tested, a hybrid algorithm combining aspects of GD and $\mathrm{CO}$ is clearly the most successful at avoiding local minima and reaching the global optimal solution, particularly in cases where a GD algorithm alone failed to identify the target parameter combination. There exists significant potential to refine and improve this form of algorithm when it is applied to more clinically realistic geometries beyond the proof of concept implementation considered here.

\section{REFERENCES}

[1] T. Krouskop, T. Wheeler, F. Kallel, B. Garra, and T. Hall, "Elastic moduli of breast and prostate tissues under compression," Ultrason. Imaging, vol. 20, pp. 260-274, 1998.

[2] A. Samani, J. Bishop, C. Luginbuhl, and D. Plewes, "Measuring the elastic modulus of ex vivo small tissue samples," Phys. Med. Biol., vol. 48, pp. 2183-2198, 2003.

[3] A. Samani and D. Plewes, "An inverse problem solution for measuring the elastic modulus of intact ex vivo breast tissue tumours," Phys. Med. Biol., vol. 52, pp. 1247-1260, 2007.

[4] S. Moore, "Better breast cancer detection," IEEE Spectr., vol. 38, no. 5, pp. 50-54, May 2001.

[5] J. B. E. A. S. K. Kerlikowske, D. Grady, and V. Ernster, "Effect of age, breast density, and family history on the sensitivity of first screening mammography," J. Amer. Med. Assoc., vol. 276, pp. 33-38, 1996.

[6] M. Kriege, C. Brekelmans, C. Boetes, P. Besnard, H. Zonderland, I. Obdeijn, R. Manoliu, T. Kok, H. Peterse, M. Tilanus-Linthorst, S. Muller, S. Meijer, J. Oosterwijk, L. Beex, R. Tollenaar, H. de Koning, E. Rutgers, and J. Klijn, "Effacy of MRI and mammography for breast cancer screening in women with familial or genetic disposition," N. Engl. J. Med., vol. 351, pp. 427-437, 2004.

[7] R. Sinkus, K. Siegmann, M. Tanter, T. Xydeas, and M. Fink, "MR elastography is capable of increasing the specificity of MR mammographyInfluence of rheology on the diagnostic gain," in Proc. 5th Int. Conf. Ultrason. Meas. Imag. Tissue Elasticity, 2006, p. 111.

[8] M. Doyley, J. Weaver, E. V. Houten, F. Kennedy, and K. Paulsen, "Thresholds for detecting and characterizing focal lesions using steady-state MR elastography," Med. Phys., vol. 30, pp. 495-504, 2003.

[9] J. Bercoff, S. Chaffai, M. Tanter, L. Sandrin, S. Catheline, M. Fink, J. Gennisson, and M. Meunier, "In vivo breast cancer detection using transient elastography," Ultrasound Med. Biol., vol. 29, pp. 1387-1396, 2003.

[10] R. Barr, "Clinical applications of a real-time elastography technique in breast imaging," in Proc. 5th Int. Conf. Ultrason. Meas. Imag. Tissue Elasticity, 2006, p. 51. 
[11] J. Carnahan and R. Sinha, "Nature's algorithms," IEEE Potentials, vol. 20, no. 2, pp. 21-24, Apr./May 2001.

[12] T. Matsuoka, S. Yamamoto, and M. Takahara, "Prediction of structures and mechanical properties of composites using a genetic algorithm and finite element method," J. Mater. Sci., vol. 36, pp. 27-33, 2001.

[13] R. Olmi, M. Bini, and S. Priori, "A genetic algorithm approach to image reconstruction in electrical impedance tomography," IEEE Trans. Evol. Comput., vol. 4, pp. 83-88, 2000.

[14] Y. Zhang, L. Hall, D. Goldgof, and S. Sarkar, "A constrained genetic approach for computing material property of elastic objects," IEEE Trans. Evol. Comput., vol. 10, pp. 341-357, 2006.

[15] R. Olmi, M. Bini, and S. Priori, "A combined FEM/genetic algorithm for vascular soft tissue elasticity estimation," Cardiovasc. Eng., vol. 6, pp. 93-102, 2006.

[16] A. Peters, A. Milsant, J. Rouzé, L. Ray, J. Chase, and E. V. Houten, "Digital image-based elasto-tmography: Proof of concept studies for surface-based mechanical property reconstruction," JSME Int. J., vol. 47, pp. 11171123, 2004.

[17] A. Peters, S. Wortmann, R. Elliott, M. Staiger, J. Chase, and E. V. Houten, "Digital image-based elasto-tomography: First experiments in surface based mechanical property estimation of gelatine phantoms," JSME Int. J., vol. 48, pp. 562-569, 2005.

[18] E. Van Houten, M. Doyley, F. Kennedy, J. Weaver, and K. Paulsen, "Initial in vivo experience with steady-state subzone-based MR elastography of the human breast," J. Magn. Reson. Imaging, vol. 17, pp. 72-85, 2003.

[19] P. Amestoy, A. Guermouche, J.-Y. L'Excellent, S. Pralet, G. Alessandrini, A. Morassi, and E. Rosset, "Hybrid scheduling for the parallel solution of linear systems," Parallel Comput., vol. 32, pp. 136-156, 2006.

[20] A. Peters, H. Uwe-Berger, J. Chase, and E. V. Houten, "Digital imagebased elasto-tomography: Non-linear mechanical property reconstruction of homogenous gelatine phantoms," Int. J. Inf. Syst. Sci., vol. 2, pp. 512$521,2006$.

[21] A. Peters, J. Chase, and E. V. Houten, "Digital image-based elastotomography: Mechanical property estimation of silicone phantoms," Mech. Biol. Eng. Comput., vol. 46, pp. 205-212, 2008.

[22] J. Holland, Adaptation in Natural and Artificial Systems. Cambridge, MA: MIT Press, 1992.

[23] S. Kirkpatrick, D. G. Jr., and M. Vecchi, "Optimization by simulated annealing," Science, vol. 220, pp. 671-680, 1983.

[24] J. Zhong, X. Hu, J. Zhang, and M. Gu, "Comparison of performance between different selection strategies on simple genetic algorithms," in Computational Intelligence for Modelling, Control and Automation. Piscataway, NJ: IEEE Press, 2005, pp. 1115-1121.

[25] N. Metropolis, A. Rosenbluth, M. R. A. Teller, and E. Teller, "Equation of state calculations by fast computing machines," J. Chem. Phys., vol. 21, pp. 1087-1092, 1953.

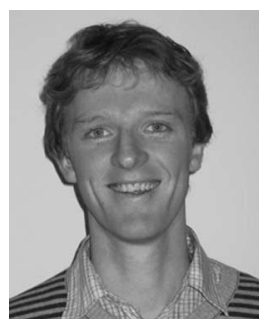

Ashton Peters was born in New Plymouth, New Zealand, in 1982. He received the Honors and Ph.D. degrees in mechanical engineering from the University of Canterbury, Christchurch, New Zealand, in 2003 and 2007, respectively.

$\mathrm{He}$ is currently a Development Engineer at Boundary Lifesciences, Inc., Christchurch, and a Visiting Researcher at the University of Canterbury, where he is engaged in the development of novel breast imaging technology.

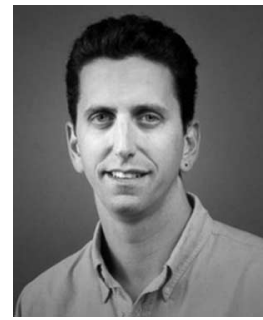

J. Geoffrey Chase received the B.S. in mechanical engineering from the Case Western Reserve University, Cleveland, OH, in 1986, and the M.S. and Ph.D. degrees from the Department of Mechanical and Civil Engineering, Stanford University, Stanford, CA, in 1991 and 1996, respectively.

He was with General Motors for six years. For five years, he was consulting and doing research in Silicon Valley, including positions at Xerox Palo Alto Research Center (PARC), GN ReSound, and Infineon Technologies AG. In 2000, he joined the University of Canterbury, Christchurch, New Zealand. His current research interests include biomedical systems and devices, structural dynamics and vibrations, dynamic systems modeling, automatic control, microelectromechanical systems (MEMS), and microfabrication and embedded systems. He is the author or coauthor of more than 280 international, refereed journal, and conference papers in these areas over the past seven years. He is the holder of ten U.S. and European patents.

Dr. Chase is a member of the International Federation on Automatic Control (IFAC) Technical Committee (IFAC TC 8.2) on Biological and Medical Systems. He is also on the Editorial Board of three journals in bioengineering and clinical medicine.

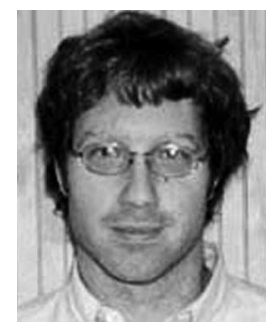

Elijah E. W. Van Houten was born in Littleton, NH, in 1974. He received the B.S. degree in mechanical engineering and the B.A. degree in music from Tufts University, Medford, MA, in 1997, and the Ph.D. degree in engineering science from Thayer School of Engineering, Dartmouth College, Hanover, $\mathrm{NH}$, in 2001.

Since 2001, he has been with the Department of Mechanical Engineering, University of Canterbury, Christchurch, New Zealand, where he is currently a Senior Lecturer in computational solid mechanics. His current research interests include computational methods and the inverse problem in elastodynamics. 